\title{
In vivo evidence of IGF-I-estrogen crosstalk in mediating the cortical bone response to mechanical strain
}

\author{
Subburaman Mohan ${ }^{1,2,3,4}$, Chetan Girijanand Bhat ${ }^{1}$, Jon E Wergedal ${ }^{1,2}$ and Chandrasekhar Kesavan ${ }^{1,2}$
}

\begin{abstract}
Although insulin-like growth factor-I (IGF-I) and estrogen signaling pathways have been shown to be involved in mediating the bone anabolic response to mechanical loading, it is not known whether these two signaling pathways crosstalk with each other in producing a skeletal response to mechanical loading. To test this, at 5 weeks of age, partial ovariectomy (pOVX) or a sham operation was performed on heterozygous IGF-I conditional knockout (H IGF-I KO) and control mice generated using a Cre-loxP approach. At 10 weeks of age, a $10 \mathrm{~N}$ axial load was applied on the right tibia of these mice for a period of 2 weeks and the left tibia was used as an internal non-non-loaded control. At the cortical site, partial estrogen loss reduced total volumetric bone mineral density (BMD) by $5 \%$ in control pOVX mice ( $P=0.05$, one-way ANOVA), but not in the H IGF-I KO pOVX mice. At the trabecular site, bone volume/total volume (BV/TV) was reduced by $5 \%-6 \%$ in both control pOVX $(P<0.05)$ and H IGF-I KO pOVX $(P=0.05)$ mice. Two weeks of mechanical loading caused a $7 \%-8 \%$ and an 11\%-13\% (P<0.05 vs. non-loaded bones) increase in cortical BMD and cortical thickness (Ct.Th), respectively, in the control sham, control pOVX and H IGF-I KO sham groups. By contrast, the magnitude of cortical BMD $(4 \%, P=0.13)$ and $\mathrm{Ct}$.Th $(6 \%, P<0.05)$ responses were reduced by $50 \%$ in the $\mathrm{H}$ IGF-I KO pOVX mice compared to the other three groups. The interaction between genotype and estrogen deficiency on the mechanical loading-induced cortical bone response was significant $(P<0.05)$ by two-way ANOVA. Two weeks of axial loading caused similar increases in trabecular BV/TV (13\%-17\%) and thickness (17\%-23\%) in all four groups of mice. In conclusion, partial loss of both estrogen and IGF-I significantly reduced cortical but not the trabecular bone response to mechanical loading, providing in vivo evidence of the above crosstalk in mediating the bone response to loading.
\end{abstract}

Bone Research (2014) 2, 14007; doi:10.1038/boneres.2014.7; Published online 29 April 2014

\section{INTRODUCTION}

Physical exercise has been used as one of the strategies to maintain bone mass and prevent bone loss in humans. Past studies using different loading models in humans and animals have demonstrated that loading stimulates bone formation while unloading decreases bone formation. ${ }^{1-5}$ In terms of how exercise increases bone formation, studies using in vitro and in vivo models, have identified involvement of multiple signaling pathways and genes. ${ }^{6-9}$ In addition to these independent pathways, one or more of these pathways may also crosstalk with each other to amplify the bone response to loading. However, the issue of whether these crosstalks significantly contribute to the bone adaptive response to mechanical strain in vivo has not been evaluated. In this study, we evaluated if insulinlike growth factor-I (IGF-I)-estrogen crosstalk contributes to the bone response to loading.

Our rationale for focusing on the IGF-I and the estrogen signaling pathway crosstalk is based on the following. By using a conditional knock out approach, we have determined that osteoblast-produced IGF-l is very important in translating the mechanical signal into anabolic effects on bone. ${ }^{10}$ Similarly, reports using estrogen receptor knockout (KO) mice have demonstrated that the loading induced bone response is diminished as a result of loss of estrogen signaling. ${ }^{11-13}$ In addition, studies using in vitro models have shown that IGF-I and estrogen may crosstalk to amplify the biological response to mechanical strain. In this

\footnotetext{
${ }^{1}$ Musculoskeletal Disease Center, VA Loma Linda Healthcare System, Loma Linda, CA, USA; ${ }^{2}$ Department of Medicine, Loma Linda University, Loma Linda, CA, USA; ${ }^{3}$ Department of Biochemistry, Loma Linda University, Loma Linda, CA, USA and ${ }^{4}$ Department of Physiology, Loma Linda University, Loma Linda, CA, USA.

Correspondence: C Kesavan (chandrasekhar.kesavan@va.gov)

Received: 12 December 2013; Revised: 4 January 2014; Accepted: 11 January 2014; Uncorrected proof published 9 April 2014
} 
regard, it has been shown that mechanical strain increases IGF-I expression in osteoblast cells, which in turn activates estrogen receptor phosphorylation via Erk1/2 activation. ${ }^{8}$ Furthermore, estrogen treatment induces proliferation via IGF-I, which in turn, enhances estrogen receptor mediated transactivation, possibly via Erk1/2, Akt and Src/Jnk activations. ${ }^{14-16}$ Although data from these in vitro studies illustrate the importance of IGF-I and estrogen crosstalk in mediating the biological response, the role of this crosstalk in relation to the bone response to loading in vivo has not been evaluated. To test the hypothesis that IGF-I-estrogen crosstalk is important in determining the amount of new bone formed in response to mechanical loading, we chose a model in which IGF-I and/or estrogen were partially depleted since the biological effect caused by partial depletion was minimal when compared to a total loss of either IGF-I or estrogen alone which completely abolished the mechanical strain effect on bone formation. ${ }^{10}$ Our rationale was that the use of heterozygous conditional IGF-I KO mice with a single copy of the IGF-I gene depleted in osteoblasts and partial ovariectomized mice with a deletion of one ovary will provide a sensitive means to evaluate the interaction between IGF-I and estrogen signaling pathways in mediating the biological response to mechanical loading. We, therefore, performed sham or partial ovariectomy (pOVX) in wild-type and heterozygous conditional IGF-I KO mice and evaluated the mechanical loading response, 5 weeks after pOVX.

\section{MATERIALS AND METHODS}

Animals

Breeding pairs of transgenic mice in which Cre recombinase is driven by the entire regulatory region of procollagen, type 1 a2 gene (Colla2-Cre) were bred with transgenic mice in which exon 4 of the IGF-I gene is flanked by the loxp gene (IGF-lox/lox) to generate $\mathrm{Cre}^{+} \mathrm{loxp}^{+/-}$ (heterozygous IGF-I KO mice) and Cre-loxp ${ }^{+/-}$(littermate control) mice as described earlier. ${ }^{10}$ A schematic representation of the study design is shown in Figure 1. The experimental procedures performed in this study were approved by the IACUC at the Jerry L. Pettis Memorial Veterans Affairs Medical Center.

\section{Genotyping}

At 3 weeks of age, DNA was extracted from tail tissue using a PUREGENE DNA Purification Kit (Gentra Systems, Inc., Minneapolis, MN, USA) and a PCR reaction was performed to identify mice with Cre recombinase and/or loxP sites as described earlier. ${ }^{10}$

\section{Ovariectomy}

To induce estrogen loss, we performed partial OVX or sham at 5 weeks of age in heterozygous IGF-I conditional knockout (H IGF-I KO) mice and IGF-I control mice. pOVX was performed as described earlier. ${ }^{17}$

\section{Axial loading}

At 10 weeks of age, the anabolic effects of exercise on both trabecular and cortical bone in these mice were evaluated using a tibia axial loading model. The apparatus and protocol for dynamically loading the mice has been adapted from previously published studies. ${ }^{1,10}$ At 10 weeks of age, a $10 \mathrm{~N}$ axial load was applied to the cortical site of tibia of sham or pOVX H IGF-I KO and control mice, respectively. The right tibia was used for loading and the left tibia was used as a contralateral non-externally loaded control. The loading was performed at 3 alternate days/week for 2 weeks. Mice were anesthetized while loading using $4 \%$ isoflurane and $2 \mathrm{~L} \cdot \mathrm{min}^{-1}$ medical oxygen. On day 15, mice were euthanized and the bones were collected and stored at $-80^{\circ} \mathrm{C}$ or in $10 \%$ formalin for further study.

\section{Micro-CT}

To measure loading induced changes in cortical and trabecular site (secondary spongiosa), micro-CT, a high resoIution tomography image system (In vivo CT40; Scanco, Medical AG, Brüttisellen Switzerland) was utilized as described earlier. ${ }^{10,18}$ Parameters such as total volume (TV, $\mathrm{mm}^{3}$ ), bone volume/total volume (BV/TV, \%), cortical thickness (Ct.Th, $\mathrm{mm}$ ), apparent density refer to bone mineral density (BMD) $\left(\mathrm{mgHA} \cdot \mathrm{ccm}^{-1}\right)$, trabecular number (Tb.N, $\mathrm{mm}^{-1}$ ), trabecular thickness (Tb.Th, $\mathrm{mm}$ ) and trabecular separation (Tb.Sp, $\mathrm{mm}$ ) were evaluated in the externally loaded right and non-externally loaded left tibia of sham or pOVX H IGF-I KO and control mice.

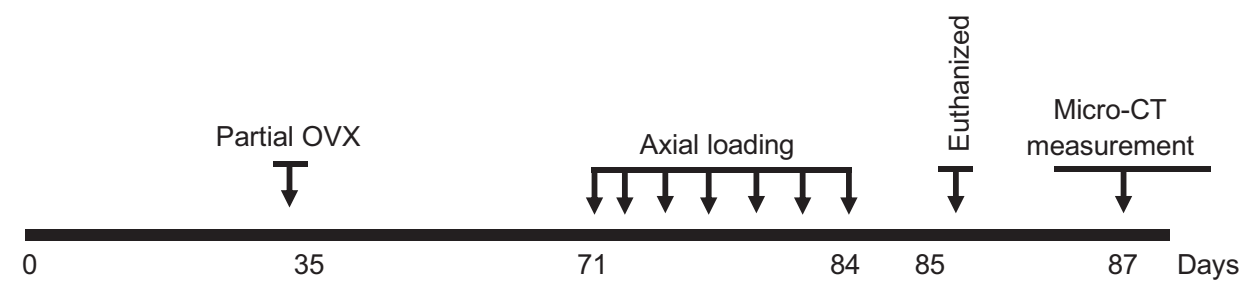

Figure 1. A schematic representation of the study design. 


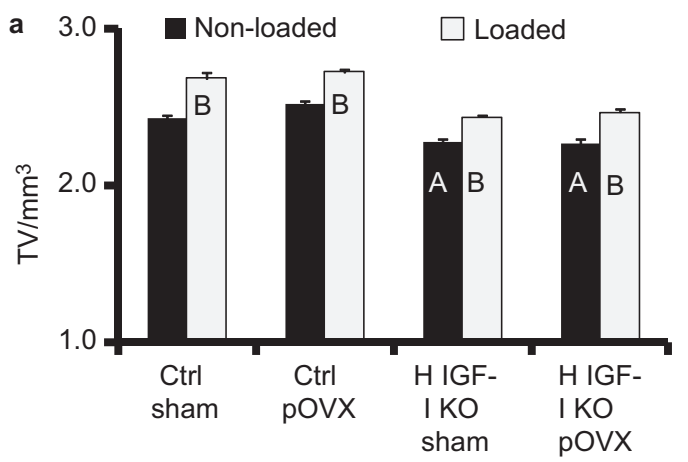

b

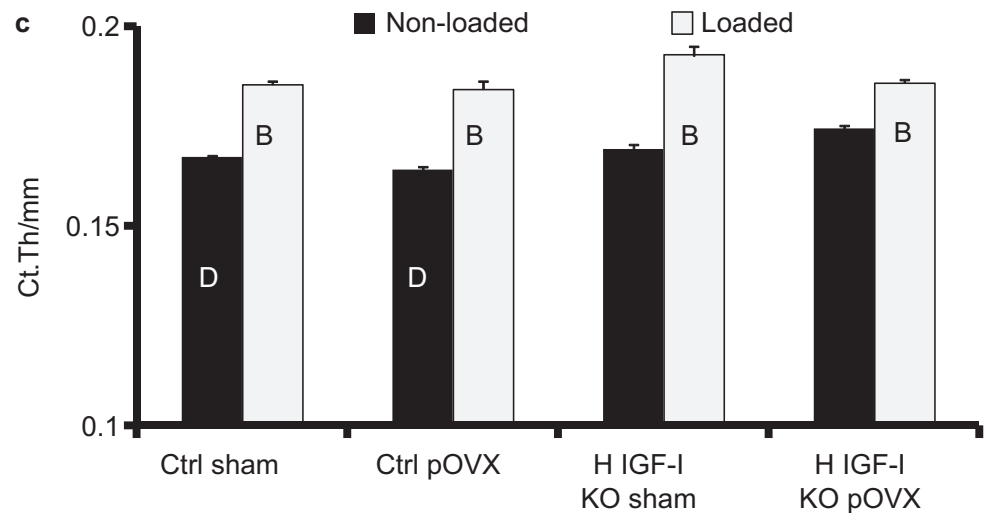

Figure 2. Micro-CT measurement of tibia cortical bone parameters (a) TV, (b) BMD and (c) Ct.Th in H IGF-I KO and control mice with sham or pOVX groups after 2 weeks of axial loading. Values are the mean \pm s.e., $N=5$ /group, ${ }^{\mathrm{A}} P<0.05 v$ s. control sham and control $\mathrm{pOVX},{ }^{\mathrm{B}} P<0.05 v$ s. non-loaded tibias, $P=0.08$ vs. control sham mice, ${ }^{C} P<0.05$ vs. H IGF-I KO sham and H IGF-I KO pOVX and ${ }^{\mathrm{D}} P=0.08$ vs. H IGF-I KO pOVX mice.

Statistical analysis

Data were presented as mean \pm s.e. t-test was used to compare basal differences in bone parameters between pOVX and sham group and for bone response to mechanical loading between non-loaded and loaded bones. Two-way ANOVA analyses were used to evaluate the basal difference and loading response between the groups.

\section{RESULTS}

Skeletal changes induced by partial estrogen loss

Cortical bone. pOVX did not affect cross sectional area at the mid diaphysis region either in the control mice or in $\mathrm{H}$ IGF-I KO mice when compared to corresponding sham groups (Figure 2a). However, total BMD was decreased by $5 \%$ in control pOVX mice when compared to control sham mice $(P=0.08)$ (Figure 2b). This decrease in BMD in the control pOVX mice, however, did not achieve statistical significance $(P=0.16)$ by two-way ANOVA. The HIGF-I KO mice with POVX did not show a decrease in BMD when compared to HIGF-I KO sham mice (Figure 2b). Twoway ANOVA analysis revealed no significant difference in the cortical thickness between the pOVX and sham groups (Figure 2c).
Trabecular bone. Control pOVX mice $(P=0.09)$ and H IGF-I KO pOVX mice $(P=0.07)$ showed a $5 \%-6 \%$ decrease in $\mathrm{BV} / \mathrm{TV}$ at the secondary spongiosa of the tibial metaphysis when compared to the corresponding sham groups (Figure 3a). Two-way ANOVA analysis revealed a significant decrease in BV/TV in the pOVX group when compared to the sham group $(P=0.02)$. Trabecular number, thickness and separation did not show any difference between the groups (Figure 3b-3d).

\section{Skeletal changes induced by partial IGF-l loss at the} cortical and trabecular site

The H IGF-I KO sham mice showed a slight increase in BMD $(P=0.05)$ and Ct.Th $(P=0.50)$ when compared to control sham at the cortical site (Figure 2). Similarly, the H IGF-I $\mathrm{KO}$ sham mice also showed slight increase in BV/TV $(P=0.10)$, Tb. $N(P=0.53)$ and Tb. Th $(P=0.48)$ at the trabecular site but was not statistically significant (Figure 3) when compared to control sham mice.

Cortical bone response to mechanical loading Two weeks of mechanical loading increased total bone volume (TV) by $7 \%-10 \%$, BMD by $7 \%-8 \%$ and cortical thickness by $10 \%-13 \%$ in the loaded tibia when compared to 

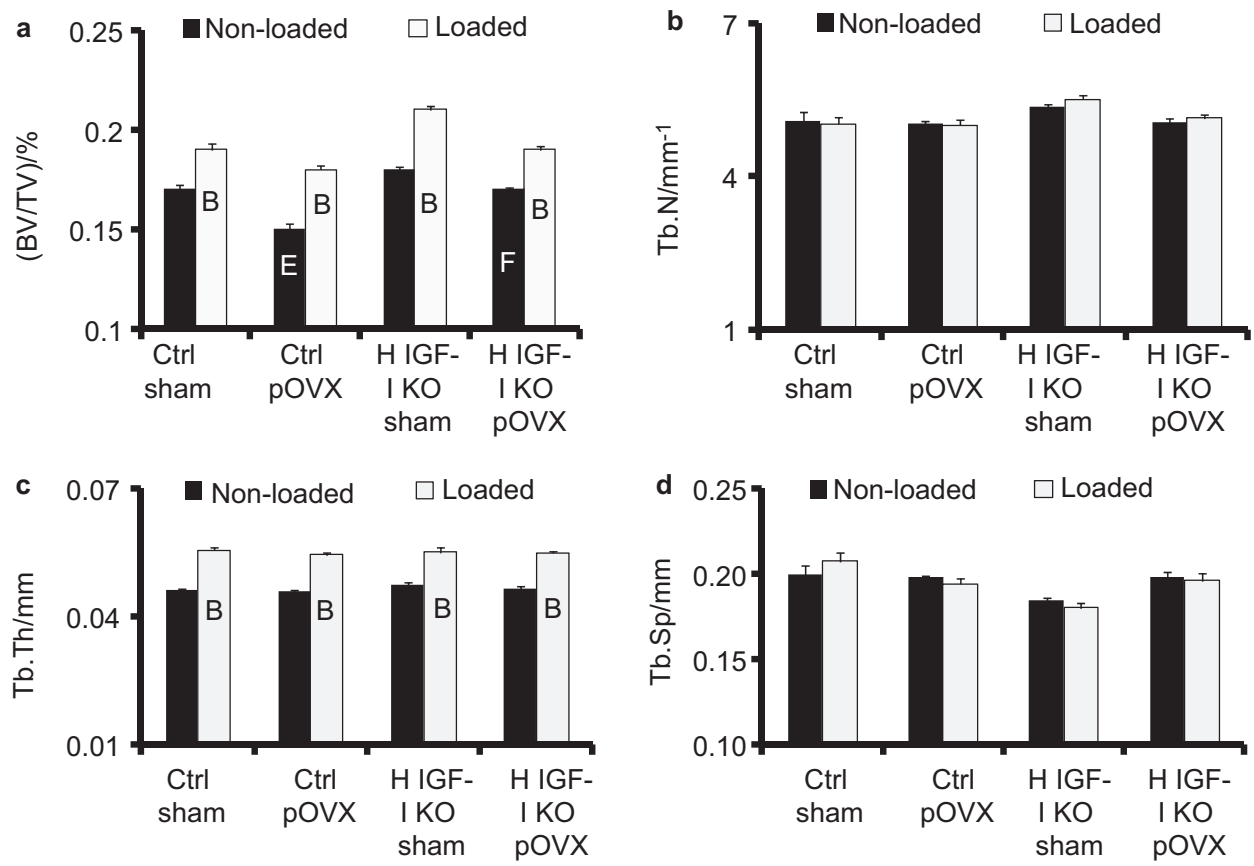

Figure 3. Micro-CT measurement of tibia trabecular bone parameters (a) BV/TV, (b) trabecular number, (c) trabecular thickness and (d) trabecular space in H IGF-I KO and control mice with sham or pOVX groups after 2 weeks of axial loading. Values are the mean \pm s.e., $N=5 /$ group, ${ }^{B} P<0.05 v s$. non-loaded tibias, ${ }^{\mathrm{E}} P<0.05$ vs. control sham and ${ }^{\mathrm{F}} \mathrm{P}=0.05$ vs. H IGF-I KO sham.

non-loaded tibia of control sham, H IGF-I KO sham and control pOVX mice (Figure 2). By contrast, in the H IGF-I $\mathrm{KO}$ mice with partial estrogen loss, TV increased by $8.8 \%$, BMD by $4 \%$ and cortical thickness by $6 \%$ in the loaded tibia when compared to non-loaded tibia after 2 weeks of mechanical loading (Figure 2). The H IGF-I KO mice with pOVX showed a reduced percent change in the bone response (BMD and Ct.Th) to mechanical loading when compared to control sham $(P=0.02)$, control pOVX $(P<0.01)$ and H IGF-I KO sham mice $(P<0.01)$.

Two-way ANOVA analysis revealed that the interaction between IGF-I and estrogen deficiency on the mechanical loading induced cortical bone response (BMD and Ct.Th) was significant ( $P=0.02)$ (Table 1).

Trabecular bone response to mechanical loading Two weeks of mechanical loading increased BV/TV, trabecular thickness and trabecular BMD in the loaded tibia by $12 \%-23 \%$ when compared to non-loaded tibia in all four groups of mice but there was no significant difference in

Table 1. Interaction analysis by two-way ANOVA between IGF-I, pOVX and mechanical loading

\begin{tabular}{llll}
\hline Bone response & Parameters & $F$ & Significance \\
\hline Cortical response & VBMD & 5.95 & 0.02 \\
$(n=20)$ & Ct.Th & 7.63 & 0.01 \\
& TV & 1.44 & 0.24 \\
Trabecular response & BV/TV & 0.63 & 0.43 \\
$(n=20)$ & Tb.N & 0.013 & 0.91 \\
& Tb.Th & 1.19 & 0.29 \\
\hline
\end{tabular}

the bone response between the groups in response to loading (Figure 3).

\section{DISCUSSION}

In our study, the control mice and H IGF-I KO mice with pOVX showed a $5 \%-6 \%$ decrease in trabecular BV/TV at the secondary spongiosa of the tibia metaphysis when compared to corresponding shams (Figure 3). This finding is consistent with previous studies that have shown decreased trabecular bone due to estrogen deficiency. ${ }^{18}$ Furthermore, two-way ANOVA analysis $(P=0.02)$ revealed that the reduced $B V / T V$ at the trabecular site in the pOVX group is due to partial estrogen loss when compared to sham mice, validating the proposed model for further study.

In this study, mice deficient for one copy of the IGF-I allele showed a slight increase in basal BMD and BV/TV at the cortical and trabecular site, respectively, when compared to the control-sham group. Consistent with this data, Bikle et al. ${ }^{19}$ have demonstrated that trabecular bone volume was increased by $23 \%$ and $88 \%$, respectively, in male and female IGF-I KO mice compared to wild-type mice. In contrast, other studies have demonstrated decreased trabecular bone volume in mice with IGF-I disruption. ${ }^{20-21}$ It remains to be determined whether the differences in trabecular bone response to IGF-I gene disruption is due to genetic background of mice used in different studies.

Previously, we have shown that the cortical bone anabolic response induced by mechanical loading is 
impaired in mice deficient for osteoblast derived IGF-I using a conditional knockout approach. ${ }^{10}$ Likewise, other groups have shown that loss of estrogen signaling via disruption of the estrogen receptor in $\mathrm{KO}$ mice resulted in a reduced loading-induced cortical bone response in mice. ${ }^{12}$ Therefore, we anticipated a reduced bone response to loading in mice with loss of partial IGF-I and estrogen signaling compared to POVX or H IGF-I KO mice if there was an active IGF-I and estrogen crosstalk in the mechanical signaling pathway. Accordingly, 2 weeks of mechanical loading at the tibia cortical site increased BMD and cortical thickness by $7 \%-13 \%$ in control sham, $\mathrm{H}$ IGF-I KO sham and the control pOVX group. By contrast, in the H IGF-I KO mice with POVX, both BMD and cortical thickness were increased only by $4 \%-6 \%$ in response to loading. Two-way ANOVA analysis $(P=0.02)$ revealed that the interaction between IGF-I and estrogen deficiency on the mechanical loading induced cortical bone response was significant (Table 1). However, in mice with partial loss of estrogen or IGF-I, the bone response to loading was similar to that of control, thus confirming an estrogen-IGF-I crosstalk in cortical bone response to mechanical strain.

In terms of mechanism, we have shown that the reduced bone forming osteoblast cell function was the cause for the impaired bone response to mechanical loading in the IGF-I CKO mice. ${ }^{10}$ Similarly, other studies also have shown that estrogen receptor (ER) primarily affects osteoblast cell function in mediating the mechanical effect on bone. ${ }^{22}$ Therefore based on this we predict that the IGF-I-estrogen crosstalk mediated mechanical effects on cortical bone is primarily through osteoblast cell; however, further study is necessary to validate this prediction.

In addition to cortical bone, we also measured loading induced changes in trabecular bone, a site more relevant to osteoporotic manifestation. As shown in Figure 3, in response to 2 weeks of axial loading, BV/TV, trabecular thickness and trabecular BMD were increased by $5 \%-23 \%$ in all four groups of mice (Figure 3). The mechanism by which axial loading increased new bone formation at the trabecular site occurs largely by increasing the thickness of existing trabeculae rather than by generating new trabeculae and this is consistent with our earlier findings. ${ }^{10}$ However, there was no significant difference in the trabecular bone response between the groups. There are several potential explanations for the lack of IGF-I and estrogen crosstalk at trabecular sites which include: (i) recent studies have shown that mice deficient for $E R \alpha$ or ER $\beta$ had no effect on the trabecular bone response to loading. Therefore, in our study, it is possible that estrogen loss may not be having any effect on the trabecular bone response to loading; (ii) the signaling pathway involved in translating the mechanical signal into an anabolic effect on bone at the trabecular site may be different from that of cortical site; (iii) we have used only one load dose in our study. Further studies with other loads as well as different amounts of IGF-I and estrogen loss are necessary to test if the trabecular bone response to mechanical loading is also influenced by estrogen-IGF-I crosstalk.

The limitations of this study are as follows. (i) Recent studies have shown that ER $\alpha$ is involved in mediating the cortical bone response to loading. While the findings of this study demonstrate that IGF-I and estrogen act together in amplifying the cortical bone response to loading, it does not address if this effect is going through $E R \alpha$ or $E R \beta$. (ii) In our study, we detected only a small reduction in trabecular bone volume in response to pOVX. It remains to be determined if the use of older mice or longer duration after pOVX would produce larger effects on trabecular bone loss. (iii) Studies in male mice are needed to determine if there is a similar crosstalk between testosterone and IGF-I in amplifying the mechanical strain effect on bone formation. (iv) In our study, pOVX was performed at 5 weeks of age in mice. Since skeletal growth is rapid during 5-10 weeks of age, it remains to be determined if the observed synergism between IGF-I and estrogen occur in the adult mice when peak BMD has been achieved. ( $v$ ) In our study conditional disruption of IGF-I gene using Coll $\alpha 2$ Cre driver would disrupt IGF-I not only in osteoblasts but also in osteocytes. However, our study cannot determine the relative contribution of osteoblast- vs. osteocytesderived IGF-I interaction with estrogen in mediating the mechanical effects on cortical bone.

In conclusion, partial loss of both estrogen and IGF-I significantly reduced cortical but not the trabecular bone response to loading, suggesting that the IGF-I-estrogen signaling interaction is involved in amplifying the anabolic effect of mechanical loading on bone formation at the cortical site. Further studies involving a larger number of mice are needed to confirm the above conclusion.

\section{Conflict of Interest}

The authors declare no conflict of interest.

\section{Acknowledgements}

This research was supported by the National Institutes of Health grant R01 AR48139 (to SM) and National Institute of Arthritis and Musculoskeletal Skin Diseases R03 grant AR056827 (to CK). The information contained in this publication does not necessarily reflect the position or the policy of the Government, and no official endorsement should be inferred. All work was performed in facilities provided by the Department of Veterans Affairs. We would like to thank Catrina, Alarcon, Joe Rung Aroon and Heather Watt for the animal work and mechanical loading experiments.

\section{References}

1 Kesavan C, Mohan S, Oberholtzer S, Wergedal JE, Baylink DJ. Mechanical loading-induced gene expression and BMD changes are different in two inbred mouse strains. J Appl Physiol 2005; 99: 1951-1957. 
2 Umemura Y, Baylink DJ, Wergedal JE, Mohan S, Srivastava AK. A time course of bone response to jump exercise in C57BL/6J mice. J Bone Miner Metab 2002; 20: 209-215.

3 Bikle DD, Sakata T, Halloran BP. The impact of skeletal unloading on bone formation. Gravit Space Biol Bull 2003; 16: 45-54.

4 Mosti MP, Kaehler N, Stunes AK, Hoff J, Syversen U. Maximal strength training in postmenopausal women with osteoporosis or osteopenia. J Strength Cond Res 2013; 27: 2879-2886.

5 Bolam KA, van Uffelen JG, Taaffe DR. The effect of physical exercise on bone density in middle-aged and older men: a systematic review. Osteoporos Int 2013; 24: 2749-2762.

6 Liedert A, Kaspar D, Blakytny R, Claes L, Ignatius A. Signal transduction pathways involved in mechanotransduction in bone cells. Biochem Biophys Res Commun 2006, 349: 1-5.

7 Boutahar N, Guignandon A, Vico L, Lafage-Proust MH. Mechanical strain on osteoblasts activates autophosphorylation of focal adhesion kinase and proline-rich tyrosine kinase 2 tyrosine sites involved in ERK activation. J Biol Chem 2004; 279: 30588-30599.

8 Lau KH, Kapur S, Kesavan C, Baylink DJ. Up-regulation of the Wnt, estrogen receptor, insulin-like growth factor-I, and bone morphogenetic protein pathways in C57BL/6J osteoblasts as opposed to $\mathrm{C} 3 \mathrm{H} / \mathrm{HeJ}$ osteoblasts in part contributes to the differential anabolic response to fluid shear. J Biol Chem 2006; 281: 9576-9588.

9 Thompson WR, Rubin CT, Rubin J. Mechanical regulation of signaling pathways in bone. Gene 2012; 503: 179-193.

10 Kesavan C, Wergedal JE, Lau KH, Mohan S. Conditional disruption of IGF-I gene in type 1alpha collagen-expressing cells shows an essential role of IGF-I in skeletal anabolic response to loading. Am J Physiol Endocrinol Metab 2010; 301: E1191-E1197.

11 Galea GL, Meakin LB, Sugiyama T et al. Estrogen receptor alpha mediates proliferation of osteoblastic cells stimulated by estrogen and mechanical strain, but their acute down-regulation of the Wnt antagonist Sost is mediated by estrogen receptor beta. J Biol Chem 2013; 288: 9035-9048.

12 Saxon LK, Galea G, Meakin L, Price J, Lanyon LE. Estrogen receptors alpha and beta have different gender-dependent effects on the adaptive responses to load bearing in cancellous and cortical bone. Endocrinology 2012; 153: 2254-2266.

13 Lee KC, Jessop H, Suswillo R, Zaman G, Lanyon LE. The adaptive response of bone to mechanical loading in female transgenic mice is deficient in the absence of oestrogen receptor-alpha and -beta. $J$ Endocrinol 2004; 182: 193-201.

14 Kato S, Endoh H, Masuhiro Y et al. Activation of the estrogen receptor through phosphorylation by mitogen-activated protein kinase. Science 1995; 270: 1491-1494.

15 Lee AV, Weng CN, Jackson JG, Yee D. Activation of estrogen receptormediated gene transcription by IGF-I in human breast cancer cells. J Endocrinol 1997; 152: 39-47.

16 Joel PB, Smith J, Sturgill TW, Fisher TL, Blenis J, Lannigan DA. pp90rsk1 regulates estrogen receptor-mediated transcription through phosphorylation of Ser-167. Mol Cell Biol 1998; 18: 1978-1984.

17 Govoni KE, Wergedal JE, Chadwick RB, Srivastava AK, Mohan S. Prepubertal OVX increases IGF-I expression and bone accretion in C57BL/6J mice. Am J Physiol Endocrinol Metab 2008; 295: E1172E1180.

18 Sugiyama T, Saxon LK, Zaman G et al. Mechanical loading enhances the anabolic effects of intermittent parathyroid hormone (1-34) on trabecular and cortical bone in mice. Bone 2008; 43: 238-248.

19 Bikle D, Majumdar S, Laib A et al. The skeletal structure of insulin-like growth factor I-deficient mice. J Bone Miner Res 2001; 16: 2320-2329.

20 Yakar S, Rosen CJ, Beamer WG et al. Circulating levels of IGF-1 directly regulate bone growth and density. J Clin Invest 2002; 110: 771-781.

21 Zhang M, Xuan S, Bouxsein ML et al. Osteoblast-specific knockout of the insulin-like growth factor (IGF) receptor gene reveals an essential role of IGF signaling in bone matrix mineralization. J Biol Chem 2002; 277: 4400544012.

22 Jessop HL, Suswillo RF, Rawlinson SC et al. Osteoblast-like cells from estrogen receptor alpha knockout mice have deficient responses to mechanical strain. J Bone Miner Res 2004; 19: 938-946.

This work is licensed under a Creative Commons AttributionNonCommercial-NoDerivs 3.0 Unported License. The images or other third party material in this article are included in the article's Creative Commons license, unless indicated otherwise in the credit line; if the material is not included under the Creative Commons license, users will need to obtain permission from the license holder to reproduce the material. To view a copy of this license, visit http:// creativecommons.org/licenses/by-nc-nd/3.0/ 